\title{
GRAVITY MODES IN ZZ CETI STARS. I. QUASI-ADIABATIC ANALYSIS OF OVERSTABILITY
}

\author{
Peter Goldreich ${ }^{1}$ AND YANQIN Wu ${ }^{1,2}$ \\ Received 1998 April 28; accepted 1998 September 3
}

\begin{abstract}
We analyze the stability of $g$-modes in white dwarfs with hydrogen envelopes. All relevant physical processes take place in the outer layer of hydrogen-rich material, which consists of a radiative layer overlaid by a convective envelope. The radiative layer contributes to mode damping, because its opacity decreases upon compression and the amplitude of the Lagrangian pressure perturbation increases outward. The convective envelope is the seat of mode excitation, because it acts as an insulating blanket with respect to the perturbed flux that enters it from below. A crucial point is that the convective motions respond to the instantaneous pulsational state. Driving exceeds damping by as much as a factor of 2 provided $\omega \tau_{c} \geq 1$, where $\omega$ is the radian frequency of the mode and $\tau_{c} \approx 4 \tau_{\mathrm{th}}$, with $\tau_{\mathrm{th}}$ being the thermal time constant evaluated at the base of the convective envelope. As a white dwarf cools, its convection zone deepens, and lower frequency modes become overstable. However, the deeper convection zone impedes the passage of flux perturbations from the base of the convection zone to the photosphere. Thus the photometric variation of a mode with constant velocity amplitude decreases. These factors account for the observed trend that longer period modes are found in cooler DA variables. Overstable modes have growth rates of order $\gamma \sim 1 /\left(n \tau_{\omega}\right)$, where $n$ is the mode's radial order and $\tau_{\omega}$ is the thermal timescale evaluated at the top of the mode's cavity. The growth time, $\gamma^{-1}$, ranges from hours for the longest period observed modes $(P \approx 20$ minutes) to thousands of years for those of shortest period $(P \approx 2$ minutes). The linear growth time probably sets the timescale for variations of mode amplitude and phase. This is consistent with observations showing that longer period modes are more variable than shorter period ones. Our investigation confirms many results obtained by Brickhill in his pioneering studies of ZZ Cetis. However, it suffers from two serious shortcomings. It is based on the quasiadiabatic approximation that strictly applies only in the limit $\omega \tau_{c} \gg 1$, and it ignores damping associated with turbulent viscosity in the convection zone. We will remove these shortcomings in future papers.
\end{abstract}

Subject headings: convection - stars: atmospheres - stars: oscillations - stars: variables: other waves

\section{INTRODUCTION}

ZZ Cetis, also called DA variables (DAVs), are variable white dwarfs with hydrogen atmospheres. Their photometric variations are associated with nonradial gravity modes ( $g$-modes); for the first conclusive proof, see Robinson, Kepler, \& Nather (1983). These stars have shallow surface convection zones overlying stably stratified interiors. As the result of gravitational settling, different elements are well separated. With increasing depth, the composition changes from hydrogen to helium, then in most cases to a mixture of carbon and oxygen. From center to surface the luminosity is carried first by electron conduction, then by radiative diffusion, and finally by convection.

Our aim is to describe the mechanism responsible for the overstability of $g$-modes in ZZ Ceti stars. This topic has received attention in the past. Initial calculations of overstable modes were presented in Dziembowski \& Koester (1981), Dolez \& Vauclair (1981), and Winget et al. (1982). These were based on the assumption that the convective flux does not respond to pulsation; this is often referred to as the frozen convection hypothesis. Because hydrogen is partially ionized in the surface layers of ZZ Ceti stars, these

\footnotetext{
${ }^{1}$ Theoretical Astrophysics, Caltech 130-33, Pasadena, CA 91125, USA; pmg@gps.caltech.edu.

2 Astronomy Unit, School of Mathematical Sciences, Queen Mary and Westfield College, Mile End Road, London E1 4NS, UK; Y.Wu@qmw.ac.uk.
}

workers attributed mode excitation to the $\kappa$-mechanism. In so doing, they ignored the fact that the thermal timescale in the layer of partial ionization is many orders of magnitude smaller than the periods of the overstable modes. Pesnell (1987) pointed out that in calculations such as those just referred to, mode excitation results from the outward decay of the perturbed radiative flux at the bottom of the convective envelope. He coined the term "convective blocking" for this excitation mechanism. ${ }^{3}$ Although convective blocking is responsible for mode excitation in the above cited references, it does not occur in the convective envelopes of $\mathrm{ZZ}$ Ceti stars. This is because the dynamic timescale for convective readjustment (i.e., convective turn-over time) in these stars is much shorter than the $g$-mode periods. Noting this, Brickhill (1983, 1990, 1991a, 1991b) assumed that convection responds instantaneously to the pulsational state. He demonstrated that this leads to a new type of mode excitation, which he referred to as convective driving. Brickhill went on to present the first physically consistent calculations of mode overstability, mode visibility, and instability strip width. Our investigation supports most of his conclusions. Additional support for convective driving is provided by Gautschy, Ludwig, \& Freytag (1996), who found overstable modes in calculations in which convection is modeled by hydrodynamic simulation.

\footnotetext{
${ }^{3}$ This mechanism was described in a general way by Cox \& Giuli (1968) and explained in more detail by Goldreich \& Keeley (1977).
} 
In this paper we elucidate the manner in which instantaneous convective adjustment promotes mode overstability. We adopt the quasiadiabatic approximation in the radiative interior. We also ignore the effects of turbulent viscosity in the convection zone. These simplifications enable us to keep our investigation analytical, although we appeal to numerically computed DA white dwarf stellar models and their eigenfunctions for guidance. The models we use are a subset of those described by Bradley (1996). They have stellar mass $M_{*}=0.6 M_{\odot}$, hydrogen layer mass 1.5 $\times 10^{-4} M_{*}$, and helium layer mass $1.5 \times 10^{-2} M_{*}$. Convection in these models is described by the ML2 version of the mixing-length theory. Fully nonadiabatic results, which require numerical computations, will be reported in a subsequent paper. These modify the details but not the principal conclusions arrived at in the present paper.

The plan of our paper is as follows: The linearized wave equation is derived in $\S 2$. In $\S 3$, we evaluate the perturbations associated with a $g$-mode in different parts of the star. We devote $\S 4$ to the derivation of a simple overstability criterion. Relevant timescales and the validity of the quasiadiabatic approximation are discussed in $\S 5$. The Appendix contains derivations of convenient scaling relations for the dispersion relation, the Wentzel-KramersBrillouin (WKB) eigenfunction, and the amplitude normalization.

\section{PREPARATIONS}

We adopt standard notation for the thermodynamic variables pressure, $p$, density, $\rho$, and temperature, $T$. Our specific entropy, $s$, is dimensionless; we measure it in units of $k_{\mathrm{B}} / m_{p}$, where $k_{\mathrm{B}}$ is the Boltzmann constant and $m_{p}$ is the proton mass. We denote the gravitational acceleration by $g$, the specific opacity by $\kappa$, the pressure scale height by $H_{p} \equiv$ $p /(g \rho)$, and the adiabatic sound speed by $c_{s}$, where $c_{s}^{2} \equiv$ $(\partial p / \partial \rho)_{s}$.

A $g$-mode in a spherical star is characterized by three eigenvalues $(n, \ell, m) ; n$ is the number of radial nodes in the radial component of the displacement vector, $\ell$ is the angular degree, and $m$ is the azimuthal separation parameter. ${ }^{4}$ Gravity modes detected in DAV stars have modest radial orders, $1 \leq n \leq 25$, and low angular degrees, $1 \leq \ell \leq 2 .{ }^{5}$ Their periods fall in the range between 100 and $1200 \mathrm{~s}$. Gravity mode periods increase with $n$ and decrease with $\ell$ (see $\S \mathrm{A} 3$ ). These properties reflect the nature of the restoring force, namely, gravity, which opposes departure of surfaces of constant density from those of constant gravitational potential.

Gravity waves propagate where $\omega$ is smaller than both the radian Lamb (acoustic) frequency, $L_{\ell}$, and the radian Brunt-Väisälä (buoyancy) frequency, $N .^{6}$ These critical frequencies are defined by $L_{\ell}^{2}=\ell(\ell+1)\left(c_{s} / r\right)^{2}$, and $N^{2} \equiv$ $g^{2}\left[d \rho / d p-(\partial \rho / \partial p)_{s, x}\right] .^{7}$ Electron degeneracy reduces the buoyancy frequency in the deep interior of a white dwarf. Consequently, the uppermost radial node of even the lowest order $g$-mode is confined to the outer few percent of the

\footnotetext{
${ }^{4}$ We ignore $m$ in this paper, since DAV's are slow rotators.

${ }^{5}$ The latter is probably an observational selection effect.

${ }^{6}$ The astronomical literature is divided on the symbol for the Lamb frequency. We follow Unno et al. (1989) and use $L_{\ell}$, while others prefer $S_{\ell}$ after $\operatorname{Cox}(1980)$.

${ }^{7}$ Here, $x$ denotes chemical composition. In regions of constant composition, $N^{2}=-g(\partial \ln \rho / \partial s)_{p} d s / d r$.
}

stellar radius. Since all of the relevant physics takes place in the region above this node, it is convenient to adopt a plane-parallel approximation with $g$ taken to be constant in both space and time. In place of the radial coordinate $r$, we use the depth $z$ below the photosphere. We neglect gravitational perturbations; because of the small fraction of the stellar mass that a mode samples, its gravitational perturbation is insignificant. A few order of magnitude relations to keep in mind are as follows: $p \sim g \rho z, H_{p} \sim z, d \ln \rho / d z \sim$ $1 / z$, and $c_{s}^{2} \sim g z$. In the convection zone, $N^{2} \sim$ $-\left(v_{\mathrm{cv}} / c_{\mathrm{s}}\right)^{2} g / z \sim-\left(v_{\mathrm{cv}} / z\right)^{2}$, where $v_{\mathrm{cv}}$ is the convective velocity. In the upper radiative interior, $N^{2} \sim g / z \sim\left(c_{s} / z\right)^{2}$. This scaling relation is compared with actual values of $N^{2}$ in Figure 1.

The linearized equations of mass and momentum conservation, augmented by the linearized, adiabatic equation of state, read $^{8}$

$$
\begin{gathered}
\frac{\delta \rho}{\rho}=-i k_{h} \xi_{h}-\frac{d \xi_{z}}{d z}, \\
\omega^{2} \xi_{h}=i k_{h}\left[\frac{p}{\rho}\left(\frac{\delta p}{p}\right)-g \xi_{z}\right], \\
\omega^{2} \xi_{z}=\frac{p}{\rho} \frac{d}{d z}\left(\frac{\delta p}{p}\right)+g\left(\frac{\delta p}{p}-\frac{\delta \rho}{\rho}-\frac{d \xi_{z}}{d z}\right), \\
\delta p=c_{s}^{2} \delta \rho .
\end{gathered}
$$

In the above equations, $\xi_{h}$ and $\xi_{z}$ represent horizontal and vertical components of the displacement vector, $\delta$ denotes a Lagrangian perturbation, and exp $i\left(k_{h} x-\omega t\right)$ describes the horizontal space and time dependence of the perturbations. The horizontal component of the propagation vector, $k_{h}$, is related to the angular degree, $\ell$, by

$$
k_{h}^{2}=\frac{\ell(\ell+1)}{R^{2}}
$$

where $R$ is the stellar radius. After some manipulation, equations (2) and (3) yield

$$
\xi_{h}=\frac{i g^{2} k_{h}}{\left(g k_{h}\right)^{2}-\omega^{4}}\left[\frac{p}{g \rho} \frac{d}{d z}\left(\frac{\delta p}{p}\right)+\left(1-\frac{\omega^{2} p}{g^{2} \rho}\right)\left(\frac{\delta p}{p}\right)\right],
$$

and

$$
\xi_{z}=\frac{-g \omega^{2}}{\left(g k_{h}\right)^{2}-\omega^{4}}\left[\frac{p}{g \rho} \frac{d}{d z}\left(\frac{\delta p}{p}\right)+\left(1-\frac{k_{h}^{2} p}{\omega^{2} \rho}\right)\left(\frac{\delta p}{p}\right)\right] .
$$

Notice that these two equations are independent of the assumption of adiabaticity.

The linear, adiabatic wave equation for the fractional, Lagrangian pressure perturbation, $\delta p / p$, follows from combining equations (1), (6), and (7):

$$
\begin{aligned}
\frac{d^{2}}{d z^{2}}\left(\frac{\delta p}{p}\right)+\frac{d}{d z} & \left(\ln \frac{p^{2}}{\rho}\right) \frac{d}{d z}\left(\frac{\delta p}{p}\right) \\
& +\left[k_{h}^{2}\left(\frac{N^{2}}{\omega^{2}}-1\right)+\left(\frac{\omega}{c_{s}}\right)^{2}\right]\left(\frac{\delta p}{p}\right)=0 .
\end{aligned}
$$

The advantages of choosing $\delta p / p$ as the dependent variable will become apparent as we proceed.

\footnotetext{
${ }^{8}$ Readers are referred to Unno et al. (1989) for detailed derivations of these equations.
} 


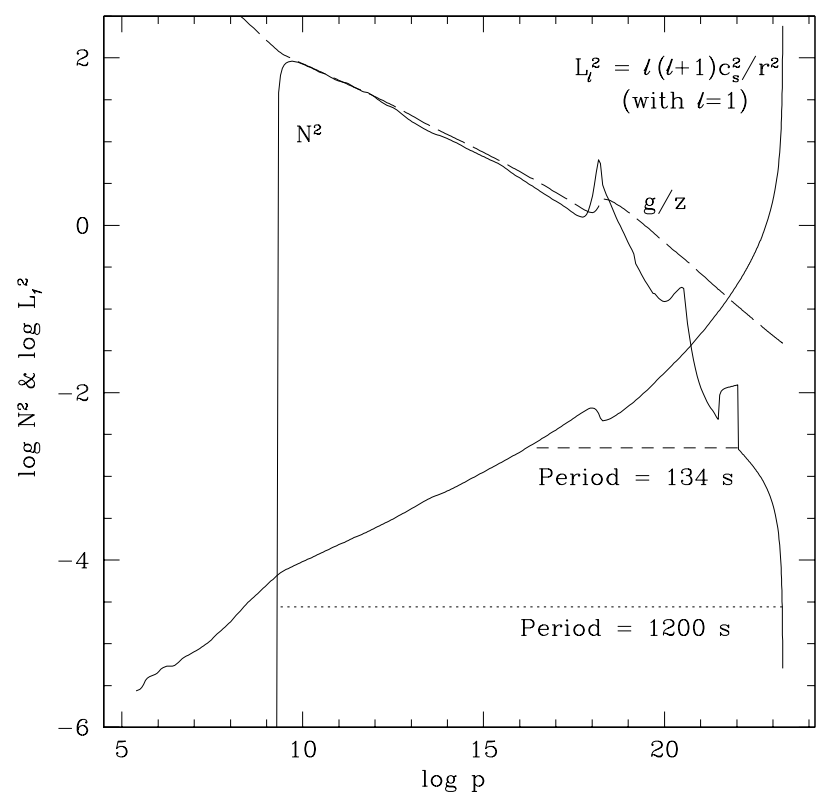

FIG. 1.-Squares of the radian Brunt-Väisälä frequency, $N^{2}$, and Lamb frequency, $L_{\ell}^{2}$, in s $\mathrm{s}^{-2}$ as functions of pressure in dynes $\mathrm{cm}^{-2}$, as calculated by Bradley for his white dwarf model of $T_{\text {eff }}=12,000 \mathrm{~K}$. The value $N^{2}$ is negative in the convection zone $(\log p \leq 9.3)$ and drops with increasing depth to near zero in the degenerate interior $(\log p \geq 23)$. The bumps in $N^{2}$ around $\log p \sim 18, \log p \sim 21$, and $\log p \sim 22$ arise from abrupt changes in chemical composition. The long-dashed line is our analytic approximation, $N^{2} \sim(g / z)$. Gravity modes with frequency $\omega$ propagate in regions where both $\omega<N$ and $\omega<L_{\ell}$. These regions are depicted by horizontal dashed lines for two $\ell=1$ modes having periods of 134 and $1200 \mathrm{~s}$, respectively. The lid on the cavity of the higher frequency mode is placed where $\omega=L_{\ell}$, while that of the lower frequency mode is located where $\omega=N$.

The Appendix is devoted to deducing the properties of $g$-modes from the wave equation (8). Here we summarize results that are needed in the main body of the paper. A $g$-mode's cavity coincides with the radial interval within which $\omega$ is smaller than both $N$ and $L_{\ell}$ (see Fig. 1). Inside this cavity the mode's velocity field is relatively incompressible. The lower boundary of each $g$-mode's cavity is set by the condition $\omega=N$. For modes of sufficiently high frequency, the relation $\omega=L_{\ell}$ is satisfied in the radiative interior, and it determines the location of the upper boundary. Otherwise, the upper boundary falls in the transition layer between the radiative interior and the convective envelope, where $\omega=N$. In this paper, we restrict consideration to modes that do not propagate immediately below the convection zone. To an order of magnitude, the condition $\omega \sim L_{\ell}$ is satisfied at a depth $z_{\omega} \sim \omega^{2} /\left(g k_{h}^{2}\right)$. If we denote the depth of the first radial node of $\delta p / p$ as $z_{1}$ and the depth at the bottom ofthe surface convection zone as $z_{b}$, then $z_{1} \sim z_{\omega}$, provided $z_{\omega} \gg z_{b}$.

The fractional Lagrangian pressure perturbation, $\delta p / p$, is almost constant in the convection zone $\left(z \leq z_{b}\right)$, and it declines smoothly to zero in the upper evanescent region $\left(z_{b} \leq z \leq z_{w}\right)$. Its envelope scales with depth in the cavity so as to maintain a constant vertical energy flux. An equal amount of energy is stored between each pair of consecutive radial nodes. For modes having $z_{\omega} \gg z_{b}$, the photospheric value of the normalized eigenfunction is given by $(\delta p / p)_{\mathrm{ph}} \sim$ $1 /\left(n \tau_{\omega} L\right)^{1 / 2}$.

\section{PERTURBATIONS ASSOCIATED WITH GRAVITY-MODE PULSATIONS}

Here we derive expressions relating various perturbation quantities associated with $g$-modes. ${ }^{9}$ These are used in $\S 4$ to evaluate the driving and damping in various parts of the star. Since theoretical details are of limited interest, we summarize selected results at the end of this section.

We start with the radiative interior and proceed outward through the convection zone to the photosphere. A new symbol, $\Delta$, is introduced to denote variations associated with a $g$-mode at a particular level, such as the photosphere, or within a particular layer, such as the convection zone. These variations are not to be confused with either Lagrangian variations, which we denote by $\delta$, or Eulerian variations.

This section is replete with thermodynamic derivatives. In most instances we take $\rho$ and $T$ as our independent variables. Unless specified otherwise, it is implicitly assumed that partial derivatives with respect to one are taken with the other held constant. We adopt the following shorthand notation: $p_{\rho} \equiv \partial \ln p / \partial \ln \rho, p_{T} \equiv \partial \ln p / \partial \ln T$, $s_{\rho} \equiv \partial s / \partial \ln \rho, s_{T} \equiv \partial s / \partial \ln T, \kappa_{\rho} \equiv \partial \ln \kappa / \partial \ln \rho$, and $\kappa_{T} \equiv$ $\partial \ln \kappa / \partial \ln T .{ }^{10}$ However, we use the symbols $\rho_{s} \equiv$ $(\partial \ln \rho / \partial s)_{p}$ and $c_{p} \equiv T(\partial s / \partial T)_{p}$. These thermodynamic quantities evaluated for a model DA white dwarf are displayed in Figure 2 for reference.

\subsection{Radiative Interior}

The radiative flux in an optically thick region obeys the diffusion equation

$$
F=\frac{4 \sigma}{3 \kappa \rho} \frac{d T^{4}}{d z},
$$

where $\sigma$ is the Stefan-Boltzmann constant. The Lagrangian perturbation of the flux takes the form

$$
\begin{aligned}
\frac{\delta F}{F}=-\left(1+\kappa_{\rho}\right) \frac{\delta \rho}{\rho}+(4- & \left.\kappa_{T}\right) \frac{\delta T}{T}-\frac{d \xi_{z}}{d z} \\
& +\left(\frac{d \ln T}{d z}\right)^{-1} \frac{d}{d z}\left(\frac{\delta T}{T}\right) .
\end{aligned}
$$

Next we express $\delta F / F$ in terms of $\delta p / p$ within the quasiadiabatic approximation. To accomplish this, we decompose $\delta \rho / \rho$ and $\delta T / T$ into adiabatic and nonadiabatic components by means of the thermodynamic identities

$$
\frac{\delta \rho}{\rho}=\frac{p}{c_{s}^{2} \rho} \frac{\delta p}{p}+\rho_{s} \delta s \approx \frac{3}{5} \frac{\delta p}{p}-\frac{\delta s}{5},
$$

and

$$
\frac{\delta T}{T}=-\frac{s_{\rho}}{s_{T}} \frac{p}{c_{s}^{2} \rho} \frac{\delta p}{p}+\frac{\delta s}{c_{p}} \approx \frac{2}{5} \frac{\delta p}{p}+\frac{\delta s}{5}
$$

\footnotetext{
${ }^{9}$ When reading this section, it is important to bear in mind that all perturbation quantities have an implicit time and horizontal space dependence of the form exp $i\left(k_{h} x-\omega t\right)$.

${ }^{10}$ The symbols $\chi_{\rho}$ and $\chi_{T}$ are generally used in the astronomical literature in place of our $p_{\rho}$ and $p_{T}$. However, the latter choices maintain the internal consistency of our notation.
} 


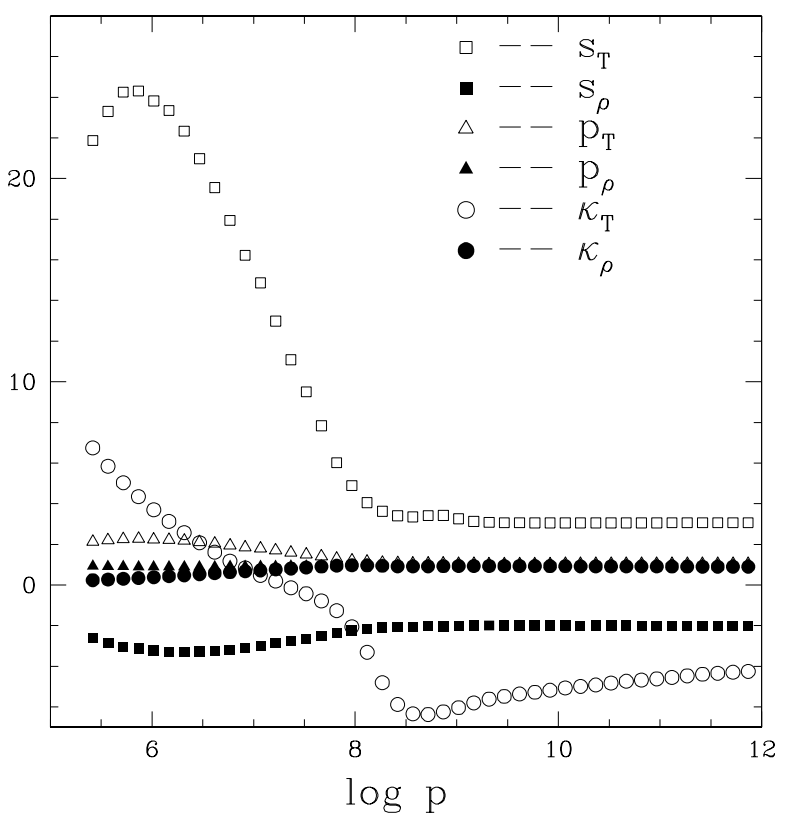

FIG. 2.- Values of various dimensionless thermodynamic quantities as functions of pressure in dynes $\mathrm{cm}^{-2}$ for Bradley's white dwarf model with $T_{\text {eff }}=12,000 \mathrm{~K}$. Here only the upper region of pure hydrogen is displayed. The convection zone extends from the surface down to $\log p \sim 9.3$. Both $s_{T}$ and $\kappa_{T}$ change significantly with depth in the upper layers where hydrogen is partially ionized. The fully ionized region has mean molecular weight $\mu=\frac{1}{2}$. Other thermodynamic quantities can be obtained from those plotted by using the following relations: $\rho_{s}=p_{T} /\left(p_{T} s_{\rho}-p_{\rho} s_{T}\right), c_{p}=$ $\left(s_{T} p_{\rho}-s_{\rho} p_{T}\right) / p_{\rho}$, and $\Gamma_{1}=c_{s}^{2} \rho / p=\left(p_{\rho} s_{T}-p_{T} s_{\rho}\right) / s_{T}$.

where the coefficients are evaluated as appropriate for fully ionized hydrogen plasma (see Fig. 2). Substitution of the adiabatic parts of these expressions into equation (10) is carried out separately for the upper evanescent layer and the $g$-mode cavity.

\subsubsection{Upper Evanescent Layer}

Within the region $z_{b} \leq z \leq z_{\omega}, \delta p / p \approx-i k_{h} \xi_{h}$, and it varies on spatial scale $z_{\omega}$ (see the Appendix). Thus equation (1) leads to

$$
\frac{d \xi_{z}}{d z} \approx \frac{\delta p}{p}-\frac{\delta \rho}{\rho} .
$$

Moreover, the term

$$
\left(\frac{d \ln T}{d z}\right)^{-1} \frac{d}{d z}\left(\frac{\delta T}{T}\right) \approx-\left(\frac{d \ln T}{d z}\right)^{-1} \frac{d}{d z}\left(\frac{s_{\rho}}{s_{T}} \frac{p}{c_{s}^{2} \rho} \frac{\delta p}{p}\right)
$$

is of order $\left(z / z_{\omega}\right)(\delta p / p)$ and can be discarded. ${ }^{11}$ Making these approximations, we arrive at

$$
\frac{\delta F}{F} \approx A \frac{\delta p}{p},
$$

where

$$
A=\frac{\left(3-3 \kappa_{\rho}-2 \kappa_{T}\right)}{5} .
$$

${ }^{11}$ As shown in Fig. 2, the factors $s_{\rho} \approx-2, s_{T} \approx 3$, and $p /\left(c_{s}^{2} \rho\right) \approx 3 / 5$ are each nearly constant in the upper part of the radiative interior.

\subsubsection{Gravity-Mode Cavity}

For $z \geq z_{\omega}$, perturbations vary on the spatial scale of $1 / k_{z}$, where $k_{z} \approx(N / \omega) k_{h}>1 / z$ is the vertical component of the wave vector as given by equation (A3). Here, the dominant contributions to $\delta F / F$ come from the last two terms in equation (10). The first transforms to

$$
\frac{d \xi_{z}}{d z} \approx-i k_{z} \frac{\delta p}{g \rho} \sim-i k_{z} z \frac{\delta p}{p},
$$

as most easily seen from equation (3). It then follows that

$$
\begin{aligned}
\frac{\delta F}{F} & \approx \frac{i k_{z} p}{g \rho} \frac{d \ln p}{d \ln T}\left(\left.\frac{\partial \ln T}{\partial \ln p}\right|_{s}-\frac{d \ln T}{d \ln p}\right) \frac{\delta p}{p} \\
& \approx \frac{i k_{z} N^{2}}{g}\left(\frac{p}{g \rho}\right)^{2} \frac{d \ln p}{d \ln T} \frac{\delta p}{p},
\end{aligned}
$$

where we have set $p_{\rho} \approx 1$ and $p_{T} \approx 1$, as is appropriate for a fully ionized plasma (see Fig. 2).

\subsection{Convective Envelope}

The net absorption of heat by the convective envelope is given by

$$
\Delta Q=\int_{\mathrm{cvz}} d z \rho \frac{k_{\mathrm{B}}}{m_{p}} T \delta s \approx \Delta s_{b} \int_{\mathrm{cvz}} d z \rho \frac{k_{\mathrm{B}}}{m_{p}} T .
$$

Here $\Delta s_{b}$ is the variation of the specific entropy evaluated at the bottom of the convection zone; it is neither a Lagrangian nor an Eulerian variation. The chain of argument leading to the second expression for $\Delta Q$ requires some discussion. At equilibrium (no pulsation), convection is so efficient that $d s / d z \ll s / z$ except in a thin superadiabatic layer just below the photosphere; the unperturbed convection zone is nearly isentropic. Moreover, the rapid response of the convective motions ensures that $d \delta s / d z \ll \delta s / z$; the vertical component of the perturbed entropy gradient is small during pulsation. Because both $d s / d z \approx 0$ and $d \delta s / d z \approx 0$, $\delta s_{b} \approx \delta s$.

To relate $\delta s$ with the flux perturbation, we treat convection by means of a crude mixing-length model. At equilibrium, the convective flux is set equal to ${ }^{12}$

$$
F \sim \alpha H_{p} v_{\mathrm{cv}} \frac{\rho k_{\mathrm{B}} T}{m_{p}} \frac{d s}{d z},
$$

where $\alpha$ is the ratio of mixing length to the pressure scale height. The convective velocity, $v_{\mathrm{cv}}$, satisfies $^{13}$

$$
v_{\mathrm{cv}}^{2} \sim-\left(\alpha H_{p}\right)^{2} g \rho_{s} \frac{d s}{d z}=-\left(\alpha H_{p} N\right)^{2} .
$$

Eliminating $d s / d z$ and solving for $v_{\mathrm{cv}}$, we find

$$
F \sim \rho v_{\mathrm{cv}}^{3} .
$$

Reversing the procedure and solving for $d s / d z$ yields

$$
s_{b}-s_{\mathrm{ph}} \equiv \int_{\mathrm{cvz}} d z \frac{d s}{d z} \approx H_{p} \frac{d s}{d z} \approx f\left(\frac{-1}{\left|\rho_{s}\right| \rho p}\right)^{1 / 3}\left(\frac{m_{p} F}{k_{\mathrm{B}} T}\right)^{2 / 3},
$$

where $s_{\mathrm{ph}}$ is the entropy at the photosphere. The dimensionless factor $f \sim \alpha^{-4 / 3}$ is of order unity. The right-hand side of

\footnotetext{
${ }^{12}$ Where the convection is efficient, $F$ is comparable to the total flux.

${ }^{13}$ Note that $\rho_{s} \equiv p_{T} /\left(p_{T} s_{\rho}-p_{\rho} s_{T}\right)<0$.
} 
equation (23) is evaluated at the photosphere, since the entropy jump is concentrated immediately below it.

We assume that the mixing-length model applies during pulsation, as is consistent with the short convective turnover time. Thus we write the variation of $s_{b}-s_{\mathrm{ph}}$ associated with a $g$-mode as

$$
\frac{\Delta\left(s_{b}-s_{\mathrm{ph}}\right)}{\left(s_{b}-s_{\mathrm{ph}}\right)}=C \frac{\Delta F_{\mathrm{ph}}}{F},
$$

where the dimensionless number $C$ is derived from equation (23) as

$$
\begin{aligned}
C= & \frac{1}{12}\left\{6+\frac{p_{T}\left(1-\kappa_{\rho}\right)+\kappa_{T}\left(1+p_{\rho}\right)}{p_{\rho}+\kappa_{\rho}}+\frac{1}{p_{T}\left(p_{\rho} s_{T}-p_{T} s_{\rho}\right)}\right. \\
& \times\left[p_{T}\left(p_{\rho T} s_{T}+p_{\rho} s_{T T}-p_{T} s_{\rho T}\right)-p_{T}^{2} s_{\rho T}-p_{T T} p_{\rho} s_{T}\right. \\
& \left.\left.+\left(\frac{p_{T}+\kappa_{T}}{p_{\rho}+\kappa_{\rho}}\right)\left(p_{T}^{2} s_{\rho \rho}-p_{T}\left(p_{\rho \rho} s_{T}+p_{\rho} s_{\rho T}\right)+p_{\rho} p_{\rho T} s_{T}\right)\right]\right\} .
\end{aligned}
$$

The dimensionless number $C$ is to be evaluated at the photosphere. In practice, only a few terms in the complicated expression for $C$ make significant contributions. In arriving at equations (24) and (25), we implicitly assume that convection carries the entire stellar flux and that $f$ does not vary during the pulsational cycle.

\subsection{Photosphere}

The photospheric temperature and pressure are determined by

$$
T \approx\left(\frac{F}{\sigma}\right)^{1 / 4}
$$

and

$$
p \approx \frac{2}{3} \frac{g}{\kappa}
$$

Provided most of the flux in the photosphere is carried by radiation, the temperature gradient is set by the equation of radiative diffusion (eq. [9]). ${ }^{14}$ Combining equations (9), (26), and (27) yields

$$
\frac{d \ln T}{d \ln p} \approx \frac{1}{8} .
$$

On the other hand, the adiabatic gradient

$$
\left.\frac{\partial \ln T}{\partial \ln p}\right|_{s}=\frac{s_{\rho}}{p_{T} s_{\rho}-p_{\rho} s_{T}} \approx \frac{1}{10},
$$

using the numbers in Figure 2. Comparing the photospheric and adiabatic temperature gradients, we find that the convection zone extends up to the photosphere for DA white $\mathrm{dwarfs}$ that lie inside the instability strip. This is confirmed by comparison with numerical models (e.g., Bradley 1996; $\mathrm{Wu}$ 1997). However, because the photospheric density is low, convection is inefficient in this region, and radiation carries the bulk of the flux.

\footnotetext{
${ }^{14}$ Applying the diffusion equation at the photosphere is a crude approximation.
}

Next we relate changes in the thermodynamic variables at the photosphere to changes in the emergent flux. Thus

$$
\frac{\Delta T}{T} \approx \frac{1}{4} \frac{\Delta F_{\mathrm{ph}}}{F}
$$

is an immediate consequence of equation (26). Expressions for $\Delta \rho / \rho$ and $\Delta p / p$ follow in a straightforward manner from the thermodynamic identity,

$$
\frac{\Delta p}{p}=p_{\rho} \frac{\Delta \rho}{\rho}+p_{T} \frac{\Delta T}{T},
$$

and from the perturbed form of equation (27),

$$
\frac{\Delta p}{p}=-\kappa_{\rho} \frac{\Delta \rho}{\rho}-\kappa_{T} \frac{\Delta T}{T} .
$$

Together, these yield

$$
\frac{\Delta \rho}{\rho} \approx-\frac{1}{4}\left(\frac{p_{T}+\kappa_{T}}{p_{\rho}+\kappa_{\rho}}\right) \frac{\Delta F_{\mathrm{ph}}}{F},
$$

and

$$
\frac{\Delta p}{p} \approx \frac{1}{4}\left(\frac{p_{T} \kappa_{\rho}-p_{\rho} \kappa_{T}}{p_{\rho}+\kappa_{\rho}}\right) \frac{\Delta F_{\mathrm{ph}}}{F} .
$$

It is then a simple step to show that

$$
\Delta s_{\mathrm{ph}} \approx B \frac{\Delta F_{\mathrm{ph}}}{F},
$$

where

$$
B=\frac{1}{4}\left[s_{T}-\left(\frac{p_{T}+\kappa_{T}}{p_{\rho}+\kappa_{\rho}}\right) s_{\rho}\right] .
$$

\subsection{Putting It All Together}

We begin by collecting a few key equations obtained in previous sections. These are then combined to determine how the entropy and flux variations in the convection zone and photosphere depend upon the pressure perturbation associated with the $g$-mode.

\subsubsection{Key Equations}

The flux perturbation entering the bottom of the convective envelope from the radiative interior satisfies (eq. [15])

$$
\frac{\Delta F_{b}}{F} \approx A\left(\frac{\delta p}{p}\right)_{b} .
$$

The photospheric entropy varies with the flux perturbation emerging from the convection zone as (eq. [35])

$$
\Delta s_{\mathrm{ph}} \approx B \frac{\Delta F_{\mathrm{ph}}}{F} .
$$

The variation in the superadiabatic entropy jump is related to this photospheric flux perturbation by (eq. [24])

$$
\Delta\left(s_{b}-s_{\mathrm{ph}}\right) \approx C \frac{\Delta F_{\mathrm{ph}}}{F} .
$$

The dimensionless numbers $A, B$, and $C$ are all positive; $A$ is evaluated at the boundary between the radiative interior and the convective envelope, whereas $B$ and $C$ are computed at the photosphere. 
The net heat variation, $\Delta Q$, and specific entropy variation, $\Delta s_{b}$, of the convective envelope are connected by equation (19):

$$
\Delta Q \approx F \tau_{b} \Delta s_{b},
$$

where the thermal time constant

$$
\tau_{b} \equiv \frac{1}{F} \int_{\mathrm{cvz}} d z \frac{\rho k_{\mathrm{B}} T}{m_{p}} \approx \frac{p_{b} z_{b}}{7 F} .
$$

The set of key equation is completed by the relation

$$
\frac{d \Delta Q}{d t}=\Delta F_{b}-\Delta F_{\mathrm{ph}} .
$$

We ignore horizontal heat transport for good reasons: transport by radiation is completely negligible, because $k_{h} z_{b} \ll 1$; turbulent diffusion acts to diminish $\Delta s_{b}$ but only at the tiny rate $k_{h}^{2} z v_{\text {cv }} \ll \omega$.

For compactness of notation, we define a new thermal time constant, $\tau_{c}$, by

$$
\tau_{c} \equiv(B+C) \tau_{b}
$$

The physical relation between $\tau_{c}$ and $\tau_{b}$ is discussed in $\S 5$. Here we merely note that $\tau_{c}$ is generally an order of magnitude or more larger than $\tau_{b}$.

\subsubsection{Implications Of Key Equations}

Taken together, the five homogeneous equations (37), (38), (39), (40), and (42) enable us to express the five quantities $\Delta s_{b}, \Delta s_{\mathrm{ph}}, \Delta F_{b} / F, \Delta F_{\mathrm{ph}} / F$, and $\Delta Q$ in terms of $(\delta p / p)_{b}{ }^{15}$ The principal results from this section read as follows:

$$
\begin{gathered}
\Delta s_{b} \approx \frac{A(B+C)}{1-i \omega \tau_{c}}\left(\frac{\delta p}{p}\right)_{b}, \\
\Delta s_{\mathrm{ph}} \approx \frac{A B}{1-i \omega \tau_{c}}\left(\frac{\delta p}{p}\right)_{b}, \\
\frac{\Delta F_{b}}{F} \approx A\left(\frac{\delta p}{p}\right)_{b}, \\
\frac{\Delta F_{\mathrm{ph}}}{F} \approx \frac{A}{1-i \omega \tau_{c}}\left(\frac{\delta p}{p}\right)_{b}, \\
\Delta Q \approx \frac{A F \tau_{c}}{1-i \omega \tau_{c}}\left(\frac{\delta p}{p}\right)_{b} .
\end{gathered}
$$

\section{DRIVING AND DAMPING}

The product of this section is the proof that a $g$-mode is linearly overstable in a ZZ Ceti star provided the convective envelope is thick enough or, more precisely, provided $\omega \tau_{c} \gtrsim 1 .^{16}$

\footnotetext{
${ }^{15}$ We include $\Delta F_{b} / F$ in this list for completeness, although it is already expressed in terms of $(\delta p / p)_{b}$ by eq. (37).

16 The qualification that $z_{\omega} \gg z_{b}$ is necessary here.
}

The time-averaged rate of change in a mode's energy,

$$
\gamma \equiv \frac{\omega}{2 \pi} \oint d t \frac{1}{E} \frac{d E}{d t}
$$

is obtained from the work integral. Useful forms for $\gamma \operatorname{are}^{17}$

$$
\begin{aligned}
\gamma & =\frac{\omega R^{2}}{2 \pi} \oint d t \int_{0}^{R} d z \rho \frac{k_{\mathrm{B}}}{m_{p}} \delta T \frac{d \delta s}{d t} \\
& =\frac{\omega L}{8 \pi^{2}} \oint d t \int_{0}^{R} d z \frac{\delta T}{T} \frac{d}{d z}\left(\frac{\delta F}{F}\right) .
\end{aligned}
$$

Regions of driving and damping are associated with positive and negative values of the above integrand. The evaluation of $\gamma$ for modes with $z_{\omega} \gg z_{b}$ is simplified by the near constancy of $\delta p / p$ for $z \ll z_{\omega}$.

\subsection{Radiative Damping}

In the upper evanescent region of the radiative interior, the perturbed flux varies in phase with the pressure perturbation, because adiabatic compression causes the opacity to decrease. ${ }^{18}$ Since $\delta p / p$ declines from close to its surface value at $z_{b}$ to zero near $z_{\omega}$ and then oscillates with rapidly declining amplitude at greater depth, the upper evanescent layer loses heat during compression and thus contributes most to mode damping.

To evaluate the radiative damping rate, it proves convenient to use the second form for $\gamma$ given by equation (50). We consider the contributions to $\gamma_{\text {rad }}$ from the upper evanescent layer, $\gamma_{r_{u}}$, and from the propagating cavity, $\gamma_{r l}$, separately.

The contribution from $z_{b} \leq z \leq z_{\omega}$, obtained with the aid of equation (12) for $\delta T / T$ and equation (46) for $\delta F / F$, reads

$$
\gamma_{r_{u}} \approx-\left(\frac{A L}{40 \pi}\right)\left(\frac{\delta p}{p}\right)_{b}^{2}+\frac{L}{40 \pi} \int_{z_{b}}^{z_{\omega}} d z\left(\frac{\delta p}{p}\right)^{2} \frac{d A}{d z} .
$$

The first term on the right-hand side generally dominates over the second one, since $\delta p / p$ declines with depth and $A$ does not vary significantly in this region.

For $z \geq z_{\omega}$, we again use equation (12) for $\delta T / T$ but now substitute equation (18) for $\Delta F / F$ to arrive at

$$
\gamma_{r_{l}} \approx \frac{-9 k_{h}^{2} L}{500 \pi g^{3} \omega^{2}} \int_{z_{\omega}}^{\infty} d z N^{4} c^{4} \frac{d \ln p}{d \ln T}\left(\frac{\delta p}{p}\right)^{2} .
$$

Appeal to equation (A8) giving the WKB envelope relation for $\delta p / p$ establishes that the integrand peaks close to $z_{\omega}$. Thus

$$
\gamma_{r_{l}} \sim-10^{-2} L\left(\frac{c N}{g}\right)_{\omega}^{4}\left(\frac{\delta p}{p}\right)_{\omega}^{2} \sim-10^{-2} L\left(\frac{\delta p}{p}\right)_{\omega}^{2},
$$

where in this context $(\delta p / p)_{\omega}$ represents the magnitude of the WKB envelope evaluated at $z_{\omega}$. Since this magnitude is significantly smaller than $(\delta p / p)_{b}$, the contribution to $\gamma$ from the $g$-mode cavity is negligible provided $z_{\omega} \gg z_{b}$.

To a fair approximation, $\gamma_{\text {rad }}$ may be set equal to the first term on the right-hand side of equation (51). Thus

$$
\gamma_{\mathrm{rad}} \approx-\left(\frac{A L}{40 \pi}\right)\left(\frac{\delta p}{p}\right)_{b}^{2} .
$$

\footnotetext{
${ }^{17}$ These assume that the eigenfunction is normalized such that $E=1$.

${ }^{18} A>0$ in eq. (46)
} 


\subsection{Convective Driving}

The perturbed flux that exits the radiative interior enters the bottom of the convection zone. There it is almost instantaneously distributed so as to maintain the vertical entropy gradient near zero. Because the convection zone gains heat during compression, it is the seat of mode driving.

To evaluate the rate of convective driving, we substitute equations (12) and (44) into the first form for $\gamma$ given by equation (50). It is apparent that the net contribution comes entirely from the adiabatic part of $\delta T / T$ (see eq. [12]). Since the integrand is strongly weighted toward the bottom of the convection zone, we evaluate all quantities there and arrive at

$$
\gamma_{\mathrm{cvz}} \approx \frac{\left(\omega \tau_{c}\right)^{2}}{1+\left(\omega \tau_{c}\right)^{2}}\left(\frac{A L}{20 \pi}\right)\left(\frac{\delta p}{p}\right)_{b}^{2} .
$$

Since $A$ is positive, so is $\gamma_{\mathrm{cvz}}$.

\subsection{Turbulent Damping}

Damping due to turbulent viscosity acting on the velocity shear in the convection zone is estimated as

$$
\gamma_{\mathrm{visc}} \approx-\frac{\omega^{2} R^{2}}{2} \int_{0}^{z_{b}} d z \rho v\left(\frac{d \xi_{h}}{d z}\right)^{2} .
$$

Here $v \sim v_{\mathrm{cv}} H_{p}$ is the turbulent viscosity, and $-i \omega d \xi_{h} / d z$ is the dominant component of velocity gradient.

For adiabatic $g$-modes in an efficient surface convection zone, there is negligible velocity shear and negligible turbulent damping. However, the mean entropy gradient in the convection zone as well as the entropy perturbation represent significant departure from isentropy. In a future paper we demonstrate that turbulent viscosity suppresses the production of velocity gradients in the convection zone to the extent that its effect on damping is negligible in comparison to radiative damping. However, turbulent damping in the region of convective overshoot at the top of the radiative interior may stabilize some long period modes near the red edge of the ZZ Ceti instability strip.

\subsection{Net Driving}

The net driving rate follows from combining equations (54) and (55):

$$
\gamma_{\mathrm{net}} \approx \frac{\left(\omega \tau_{c}\right)^{2}-1}{\left(\omega \tau_{c}\right)^{2}+1}\left(\frac{A L}{40 \pi}\right)\left(\frac{\delta p}{p}\right)_{b}^{2} .
$$

Driving exceeds damping by a factor of 2 in the limit $\omega \tau_{c} \gg$ 1. Substitution of the normalization relation given by equation (A28) for $\delta p / p$ yields the more revealing form

$$
\gamma_{\mathrm{net}} \sim \frac{\left(\omega \tau_{c}\right)^{2}-1}{\left(\omega \tau_{c}\right)^{2}+1}\left(\frac{1}{n \tau_{\omega}}\right)
$$

Overstability occurs if $\omega \tau_{c}>1{ }^{19}$ Following Brickhill, we refer to this excitation mechanism as convective driving.

\section{DISCUSSION}

\subsection{Timescales}

Three timescales are relevant for convective driving in DAVs. The first is the period of an overstable $g$-mode, $P=2 \pi / \omega$, which is typically of order a few hundred s. The second is the dynamical time constant, $t_{\mathrm{cv}} \sim H_{p} / v_{\mathrm{cv}}$, on which convective motions respond to perturbations; $t_{\mathrm{cv}} \leq$ $1 \mathrm{~s}$ throughout the convection zones of even the coolest $\mathrm{ZZ}$ Cetis. This is why the convective motions adjust to the instantaneous pulsational state. The third is the thermal time constant, $\tau_{c}$, during which the convection zone can bottle up flux perturbations that enter it from below.

Given the central role of $\tau_{c}$, we elaborate on its relation both to $t_{\mathrm{cv}}$ and to the more conventional definition of thermal time constant, $\tau_{\mathrm{th}}$, at depth $z$. The latter is the heat capacity of the material above that depth divided by the luminosity. In a plane-parallel, fully ionized atmosphere this is equivalent to

$$
\tau_{\mathrm{th}} \equiv \frac{1}{F} \int_{0}^{z} d z c_{p} \frac{\rho k_{\mathrm{B}}}{m_{p} T} \approx \frac{5 p z}{7 F} .
$$

Appeal to equation (22) establishes that inside the convection zone

$$
\frac{t_{\mathrm{cv}}}{\tau_{\mathrm{th}}} \sim\left(\frac{v_{\mathrm{cv}}}{c_{s}}\right)^{2} \ll 1 .
$$

Now $\tau_{c} \equiv(B+C) \tau_{b}$, where $\tau_{b}$ is defined by equation (41). To the extent that $c_{p} \approx 5$ is constant in the convection zone, $\tau_{b} \approx \tau_{\mathrm{th}} / 5$, where the latter is evaluated at $z_{b}$.

Next we address the relation between $\tau_{c}$ and $\tau_{b}$. Here we are concerned with the relatively large value of $B+C$, typically about 20 for DAVs. ${ }^{20}$ Recall from equations (44) and (47) that

$$
\frac{\delta F_{\mathrm{ph}}}{F} \approx \frac{\delta s_{b}}{B+C}
$$

So the photosphere and the superadiabatic layer add an insulating blanket on top of the convection zone. The large value of $B$ follows because the photospheres of DAVs are composed of lightly ionized hydrogen. In this state, the values of $\kappa_{T}$ and $s_{T}$ are both large and positive; typical values in the middle of the instability strip are $\kappa_{T} \approx 6$ and $s_{T} \approx 24$. The large and positive $\kappa_{T}$ arises because the population of hydrogen atoms in excited states that the ambient radiation field can photoionize increases exponentially with increasing $T$. The large and positive $s_{T}$ occurs because the ionization fraction increases exponentially with increasing $T$ and a free electron contributes much larger entropy than a bound one. The large and positive value of $C$ reflects the increase in entropy gradient that accompanies an increase in convective flux. It is obtained from mixing-length theory with an unperturbed mixing length.

\subsection{Validity of the Quasiadiabatic Approximation}

The validity of the quasiadiabatic approximation requires that the nonadiabatic parts of the expressions for $\delta \rho / \rho$ and $\delta T / T$, as given by equations (11) and (12), be small in comparison to the adiabatic parts. Thus the ratio

$$
\mathscr{R}_{\mathrm{na}} \equiv \frac{\delta s}{\delta p / p}
$$

is a quantitative measure of nonadiabaticity. We estimate $\mathscr{R}_{\mathrm{na}}$ for the radiative interior and the convection zone.

${ }^{20}$ In our models, $B$ and $C$ have comparable values.

\footnotetext{
${ }^{19}$ In the quasiadiabatic limit for modes with $z_{\omega} \gg z_{b}$.
} 
We calculate $\delta s$ in the radiative interior from

$$
\delta s \approx \frac{i F}{\omega} \frac{m_{p}}{\rho k_{\mathrm{B}} T} \frac{d}{d z}\left(\frac{\delta F}{F}\right) .
$$

In the upper evanescent layer, $z_{b}<z<z_{\omega}$, this leads to

$$
\left|\mathscr{R}_{\text {na }}\right| \sim \frac{1}{\omega \tau_{\text {th }}},
$$

whereas in the propagating cavity, $z>z_{\omega}$, we find

$$
\left|\mathscr{R}_{\text {na }}\right| \sim \frac{1}{\omega \tau_{\text {th }}}\left(\frac{z}{z_{\omega}}\right) .
$$

Nonadiabatic effects in the radiative interior are maximal at $z=z_{b}$, since $\tau_{\mathrm{th}} / z$ increases with depth.

The requirement $\omega \tau_{\text {th }} \gg 1$ for the validity of the quasiadiabatic approximation severely limits the applicability of the current investigation. The perturbed flux at the photosphere is related to that at the bottom of the convection zone by

$$
\frac{\Delta F_{\mathrm{ph}}}{F} \approx \frac{1}{1-i \omega \tau_{c}} \frac{\Delta F_{b}}{F} .
$$

Since $\tau_{c}$ is approximately 4 times larger than $\tau_{\text {th }}$ at $z_{b}$, so modes with $\omega \tau_{c}$ of order unity do not satisfy the quasiadiabatic assumption. Modes with $\omega \tau_{c} \gg 1$ are likely to exhibit small photometric variations. However, this may not render them undetectable, because their horizontal velocity perturbations pass undiminished through the convection zone.

\subsection{Brickhill's Papers}

Our investigation is closely related to studies of $\mathrm{ZZ}$ Cetis by Brickhill (1983, 1990, 1991a). Brickhill recognized that the convective flux must respond to the instantaneous pulsational state. To determine the manner in which the convection zone changes during a pulsational cycle, he compared equilibrium stellar models covering a narrow range of effective temperature. Brickhill provided a physical description of convective driving and obtained an overstability criterion equivalent to ours. ${ }^{21}$ Moreover, he recognized that the convection zone reduces the perturbed flux and delays its phase.

Our excuses for revisiting this topic are that Brickhill's papers are not widely appreciated, that our approach is different from his, and that our paper provides the foundation for future papers that will examine issues beyond those he treated.

We are indebted to Bradley for supplying us with models of DA white dwarfs. We thank an anonymous referee for constructive comments. Financial support for this research was provided by NSF grant 94-14232.

\footnotetext{
${ }^{21}$ Our time constant $\tau_{c}$ is equivalent to the quantity $D$, which Brickhill (1983) defined in eq. (9) of that paper.
}

\section{APPENDIX}

\section{SCALING RELATIONS FOR GRAVITY MODES}

The Appendix contains derivations of a number of scaling relations appropriate to $g$-modes in ZZ Cetis. These relations are applied in the main text and in our subsequent papers on white dwarf pulsations. We obtain approximate expressions for dispersion relations, eigenfunctions, and normalization constants.

\section{A1. PROPERTIES OF GRAVITY WAVES}

Substituting the WKB Ansatz,

$$
\frac{\delta p}{p}=\mathscr{A} \exp \left(i \int^{z} d z k_{z}\right),
$$

into the wave equation (8) and retaining terms of leading order in $k_{z} H_{p} \gg 1$, we obtain the dispersion relation

$$
k_{z}^{2} \approx \frac{\left(N^{2}-\omega^{2}\right)\left(k_{h}^{2} c_{s}^{2}-\omega^{2}\right)}{c_{s}^{2} \omega^{2}},
$$

where $k_{h} c_{s}$ is the plane-parallel equivalent of the Lamb frequency, $L_{\ell}$.

Gravity waves propagate in regions where $\omega$ is smaller than both $N$ and $L_{\ell}$. Far from turning points, their WKB dispersion relation simplifies to

$$
k_{z} \approx \pm \frac{N}{\omega} k_{h} .
$$

The vertical component of their group velocity is given by

$$
v_{g z} \equiv \frac{\partial \omega}{\partial k_{z}} \approx-\frac{\omega}{k_{z}} \approx \pm \frac{\omega^{2}}{N k_{h}} .
$$



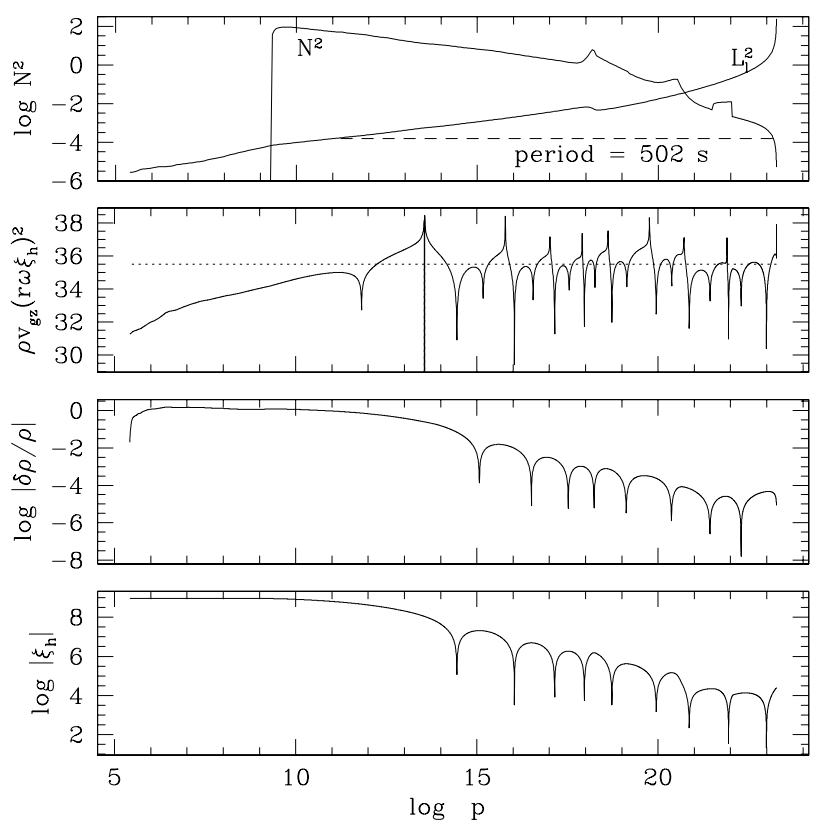

Fig. 3.-Structure of a mode with $n=9, \ell=1$, and a period of $502 \mathrm{~s}$, as a function of pressure in Bradley's model with $T_{\text {eff }}=12,000 \mathrm{~K}$. The top panel, which is similar to Fig. 1, illustrates how the mode cavity (dashed line) is formed. For this mode $z_{\omega}>z_{b}$. The WKB luminosity measured in ergs $\mathrm{s}^{-1}$ is plotted in the second panel. It is constant inside the cavity and decays outside it. The compositional transitions have minimal effects on this mode. The lower two panels display the depth dependences for the dimensionless $\delta \rho / \rho$ and $\xi_{h}$ measured in $\mathrm{cm}$. The numerical values in this figure come from setting $\xi_{h}=R$ at the photosphere.

Terms of next highest order in $k_{z} H_{p}$ yield the WKB amplitude relation for gravity waves:

$$
\mathscr{A}^{2} \propto \frac{\rho}{k_{z} p^{2}} \propto \frac{\rho}{N p^{2}} .
$$

The amplitude relation expresses the conservation of the vertical flux of wave action. This may be confirmed directly by means of an alternate derivation. A straightforward manipulation of the linear perturbation equations yields the quadratic conservation law

$$
\frac{\partial}{\partial t}\left(\frac{\rho}{2}\left|\frac{\partial \xi}{\partial t}\right|^{2}+\frac{\left(p^{\prime}\right)^{2}}{2 \rho c_{s}^{2}}+\frac{\rho}{2} N^{2} \xi_{z}^{2}\right)=-\nabla \cdot\left(p^{\prime} \frac{\partial \xi}{\partial t}\right),
$$

where $p^{\prime} \equiv \delta p-g \rho \xi_{z}$ is the Eulerian pressure perturbation. We identify $\mathscr{F} \equiv p^{\prime}(\partial \xi / \partial t)$ as the quadratic energy flux. The time-averaged magnitude of the vertical component of $\mathscr{F}$, computed to lowest order in $\left(k_{z} H_{p}\right)^{-1}$ with the aid of equations (6), (7), and (A3), is found to be

$$
\mathscr{F} \approx \frac{\omega^{2}}{g k_{h}} \frac{N p^{2}}{g \rho}\left(\frac{\delta p}{p}\right)^{2},
$$

which is in accord with the WKB amplitude relation (eq. [A5]). Yet another way to look at the energy flux is as the energy density transported by the group velocity. The energy density may be approximated as $\omega^{2} \rho \xi_{h}^{2}$, since kinetic energy contributes half the time-averaged total energy density, and $\left|\xi_{h}\right| \gg\left|\xi_{z}\right|$. Application of equations (6), (A3), and (A4) yields

$$
\mathscr{F} \approx \omega^{2} \rho \xi_{h}^{2} v_{g z} \approx \frac{\omega^{2}}{g k_{h}} \frac{N p^{2}}{g \rho}\left(\frac{\delta p}{p}\right)^{2}
$$

which reproduces the result for $\mathscr{F}$ given by equation (A7).

In a spherical star, the conserved quantity is the WKB luminosity, $\mathscr{L} \equiv 4 \pi r^{2} \mathscr{F}$. Figure 3 displays the depth dependences of $\delta \rho / \rho$ (representative of $\delta p / p)$ and $\mathscr{L}$ for a typical $g$-mode.

\section{A2. PROPERTIES OF GRAVITY MODES}

\section{A2.1. Inside the Propagating Cavity}

The Lamb frequency, $L_{\ell}$, decreases with increasing $r$, whereas the Brunt-Väisälä frequency, $N$, increases until it drops abruptly just below the convection zone and becomes imaginary within it (Fig. 1). Compositional transitions may be exceptions to these trends. The WKB approximation is violated if these transitions are sharp on the scale of the radial wavelength. When these are located in regions of propagation, they are best viewed as separating linked cavities. 
We divide a star's $g$-modes into two classes, high- and low-frequency modes. The former have cavities bounded from above at $z_{\omega}$, where $\omega=L_{\ell} \approx k_{h} c_{s}$ and $z_{\omega}>z_{b}$. The latter propagate until just below the convection zone, where $\omega=N$. The floor of the propagating cavity is set by $\omega=N$ for all modes. In this paper we are exclusively concerned with high-frequency modes. Taking $c_{s} \sim(g z)^{1 / 2}$, we find

$$
z_{\omega} \sim \frac{\omega^{2}}{g k_{h}^{2}} .
$$

Moreover, $N \sim(g / z)^{1 / 2} \sim c_{s} / z$ implies $k_{z} z_{\omega} \sim 1$ at $z \sim z_{\omega}$. Thus $z_{\omega}$ is to be identified with $z_{1}$, the uppermost node of $\delta p / p$. More generally,

$$
k_{z} \sim \frac{1}{\left(z_{\omega} z\right)^{1 / 2}}
$$

for $z>z_{\omega}$.

Expressions for $\xi_{h}$ and $\xi_{z}$ are given by equations (6) and (7). To leading order in $k_{z} H_{p}$, these reduce to

$$
\xi_{h} \approx-\frac{k_{z}}{k_{h}} \frac{p}{g \rho}\left(\frac{\delta p}{p}\right)
$$

and

$$
\xi_{z} \approx \frac{p}{g \rho}\left(\frac{\delta p}{p}\right) .
$$

Where $N / \omega \gg 1$, we have $k_{z} / k_{h} \gg 1$, which implies $\left|\xi_{h} / \xi_{z}\right| \gg 1$. In these regions $g$-modes are characterized by nearly horizontal motions. Equations (A11) and (A12) imply that $\delta \rho / \rho=-i k_{h} \xi_{h}-d \xi_{z} / d z$ vanishes to leading order in $\left(k_{z} H_{p}\right) \ll 1$. Thus $g$-modes are relatively incompressible within their cavities. A more precise estimate, obtained from equation (A11), is $\delta \rho / \rho \sim i k_{h} \xi_{h}\left(z_{\omega} / z\right)^{1 / 2}$.

\section{A2.2. Upper Evanescent Layer}

For the purpose of this discussion, we pretend that both $\rho \rightarrow 0$ and $p \rightarrow 0$ as $z \rightarrow 0$. Then $z=0$ is a singular point of the wave equation (8). Since the physical solution is regular at $z=0$,

$$
\frac{d}{d z}\left[\ln \left(\frac{\delta p}{p}\right)\right] \sim-\left(\frac{N}{\omega}\right)^{2} k_{h}^{2} z
$$

In the convection zone, where $N^{2} \approx-\left(v_{\mathrm{cv}} / z\right)^{2}$,

$$
\frac{d}{d z}\left[\ln \left(\frac{\delta p}{p}\right)\right] \sim\left(\frac{v_{\mathrm{cv}}}{c_{s}}\right)^{2} \frac{1}{z_{\omega}},
$$

and at the top of the radiative interior, where $N^{2} \sim\left(c_{s} / z\right)^{2}$,

$$
\frac{d}{d z}\left[\ln \left(\frac{\delta p}{p}\right)\right] \sim-\frac{1}{z_{\omega}} .
$$

We see that $\delta p / p$ is nearly constant for $z \ll z_{\omega}$ in the upper evanescent layer. That is why we chose to use it as the dependent variable in the wave equation.

Taking into account the near constancy of $\delta p / p$, equations (6) and (7) imply that, to leading order in $z / z_{\omega}$,

$$
\xi_{h} \approx \frac{i}{k_{h}}\left(\frac{\delta p}{p}\right)
$$

and

$$
\xi_{z} \approx-\frac{\omega^{2}}{g k_{h}^{2}}\left(\frac{\delta p}{p}\right) .
$$

Thus both components of the displacement vector are nearly constant for $z \ll z_{\omega}$. The behavior of $\delta \rho / \rho$ is more subtle; in principle, it could vary on scale $z$ should $p /\left(c_{s}^{2} \rho\right)$ do so. However, in practice, $p /\left(c_{s}^{2} \rho\right)$ exhibits only mild depth variations. Thus equation (A16) yields

$$
\frac{\delta \rho}{\rho} \sim-i k_{h} \xi_{h} .
$$

Equation (A18) shows that the relative incompressibility that characterizes propagating $g$-modes does not extend to their evanescent tails. 

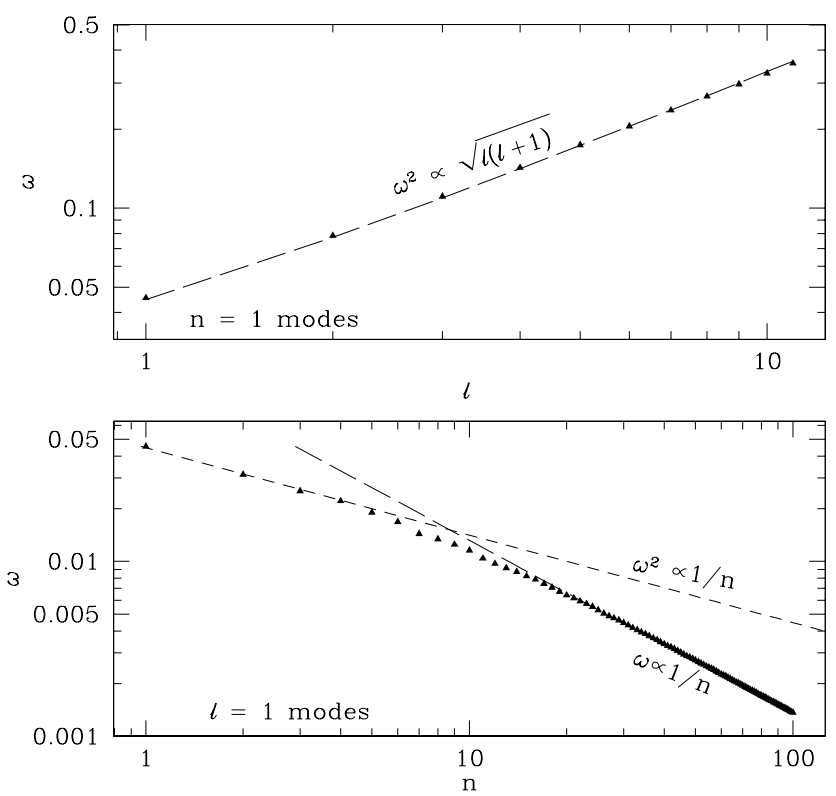

FIG. 4.- Radian frequencies of $g$-modes in $\mathrm{s}^{-1}$ as functions of radial order, $n$, and spherical degree, $\ell$. The eigenvalues are computed using Bradley's white dwarf model with $T_{\text {eff }}=12,000 \mathrm{~K}$. The upper panel is for $n=1$ modes with various angular degrees, while the lower panel is for $\ell=1$ modes of different radial orders. The global dispersion relations given by eqs. (A20) and (A21) fit well for modes of low and high orders, respectively.

\section{A3. GLOBAL DISPERSION RELATION}

The global dispersion relation is obtained from

$$
n \pi \approx \int_{z_{1}}^{z_{l}} d z k_{z},
$$

where $z_{l}$ is the lower boundary of the mode cavity. Carrying out the integration for a polytropic atmosphere where $N^{2} \sim g / z$, using $z_{1} \sim z_{\omega}$ and equation (A3), we obtain the proportionality

$$
\omega^{2} \propto \frac{k_{h}}{n},
$$

which provides a satisfactory fit to the high-frequency modes in DAV white dwarfs. However, low-frequency modes penetrate deeply into the interior, where the approximation $N \sim(\mathrm{g} / z)^{1 / 2}$ fails because of electron degeneracy (see Fig. 1). As a result of the steep drop in $N, z_{l}$ is nearly independent of $\omega$ for $\omega \leq 10^{-2} \mathrm{~s}^{-1}$. This steepens the dependence of $\omega$ on $n$ in such a way that

$$
\omega \propto \frac{k_{h}}{n} .
$$

These scalings are validated by Figure 4, which displays eigenfrequencies for $g$-modes of various $n$ and $\ell$ computed for Bradley's white dwarf model, with $T_{\text {eff }}=12,000 \mathrm{~K}$. In particular, the frequencies of the $\ell=1 g$-modes vary $\propto n^{-1 / 2}$ for $n \leq 6$ and $\propto n^{-1}$ for $n \geq 12$. We adopt these empirical laws in our analytical studies.

\section{A4. NORMALIZATION OF EIGENFUNCTIONS}

We conform to standard practice (Unno, Osaki, \& Ando 1989) and set

$$
\frac{\omega^{2}}{2} \int_{0}^{R} d r r^{2} \rho\left(\xi_{h}^{2}+\xi_{z}^{2}\right)=1 .
$$

The conservation of the WKB energy flux implies that the region between each pair of neighboring radial nodes contributes an equal amount to the above integral.

To achieve a simple analytic result, we take advantage of the following: $\left|\xi_{h}\right| \gg\left|\xi_{z}\right|,\left.\xi_{h} \sim \xi_{h}\right|_{\text {ph }}$ for $z \ll z_{\omega}$, and the envelope of $\rho v_{g z} \xi_{h}^{2} \approx$ constant for $z \gg z_{\omega}$ (see eq. [A8]). This enables us to write

$$
\left.\frac{\omega^{2}}{2} R^{2} \xi_{h}^{2}\right|_{\mathrm{ph}}\left(\rho v_{g z}\right)_{\omega} \int \frac{d z}{v_{g z}} \approx 1,
$$

where the subscript $\omega$ stands for quantities evaluated at $z_{\omega}$. Using

$$
\int_{0}^{R} \frac{d z}{v_{g z}} \approx \frac{\pi n}{\omega},
$$



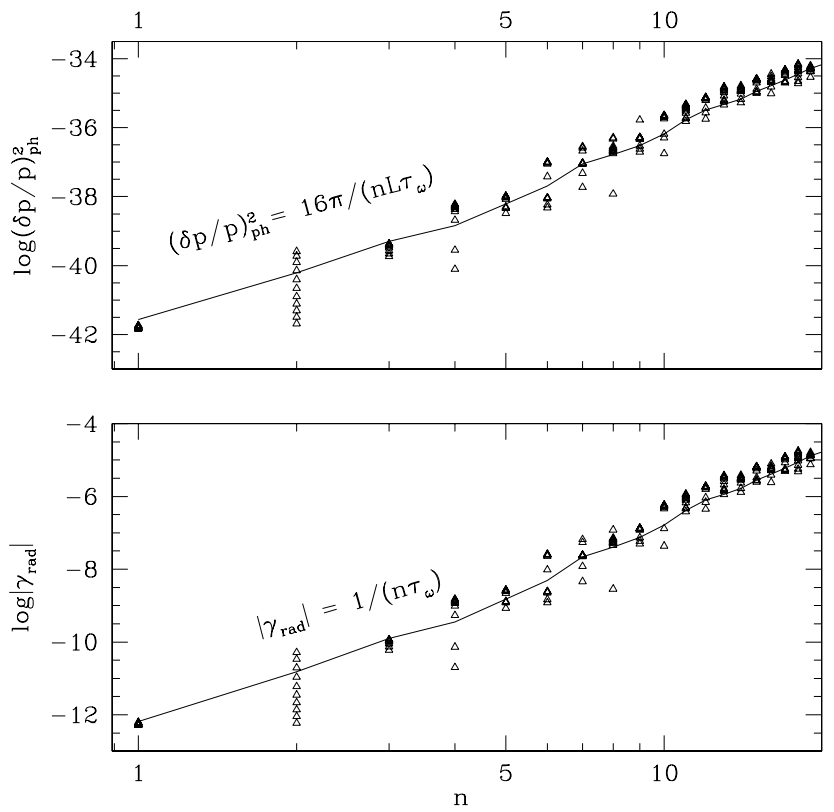

FIG. 5.-Normalized surface amplitudes and radiative damping rates for $g$-modes in white dwarfs. We compare numerical values obtained using Bradley's model with $T_{\text {eff }}=12,000 \mathrm{~K}$ with analytic estimates. Both properties depend much more strongly on $n$ than on $\ell$, hence the choice of $n$ as the abscissa. The upper panel plots as triangles numerical values of $(\delta \rho / \rho)_{\mathrm{ph}}^{2}$ in $\mathrm{g}^{-1} \mathrm{~cm}^{-2} \mathrm{~s}^{2}$ for modes with $\ell=1$ to 10 , with the solid line representing the analytical estimate from eq. (A28) for modes with $z_{\omega} \gg z_{b}$, where $z_{\omega}$ is chosen to be $3 \pi \omega^{2} /\left(2 g k_{h}^{2}\right)$. The lower panel displays numerical radiative damping rates as triangles, together with the analytic estimate from eq. (54) as a solid line.

we arrive at

$$
\left(\rho v_{g z}\right)_{\omega}=\left(\frac{\omega^{2} \rho}{k_{h} N}\right)_{\omega} \sim\left(\frac{\omega^{2} p z}{k_{h} c_{s}^{3}}\right)_{\omega} \sim \frac{k_{h}^{2} F \tau_{\omega}}{\omega}
$$

where $F$ is the stellar flux and

$$
\tau_{\omega} \approx\left(\frac{p z}{F}\right)_{\omega}
$$

is the thermal time scale at $z_{\omega}$.

Putting these relations together, we obtain

$$
\left.k_{h}^{2} \xi_{h}^{2}\right|_{\mathrm{ph}} \sim \frac{1}{n \tau_{\omega} L},
$$

where $L=4 \pi R^{2} F$ is the stellar luminosity. Equation (A16) then implies

$$
\left(\frac{\delta p}{p}\right)_{\mathrm{ph}}^{2} \sim\left(\frac{\delta \rho}{\rho}\right)_{\mathrm{ph}}^{2} \sim \frac{1}{n \tau_{\omega} L} .
$$

This is an appropriate normalization formula for modes having $z_{\omega} \gg z_{b}$. The scaling relations for the mode normalization given by equation (A28) and the damping rate given by equation (54) are compared with numerical results in Figure 5.

Bradley, P. A. 1996, ApJ, 468, 350

Brickhill, A. J. 1983, MNRAS, 204, 537

1990, MNRAS, 246, 510

1991a, MNRAS, 251,673

1991b, MNRAS, 252, 334

Cox, J. P. 1980, Theory of Stellar Pulsations (Princeton: Princeton University Press)

Cox, J. P., \& Giuli, R. T. 1968, Principles of Stellar Structure (New York: Gordon and Breach)

Dolez, N., \& Vauclair, G. 1981, A\&A, 102, 375

\section{REFERENCES}

Dziembowski, W., \& Koester, D. 1981, A\&A, 97, 16

Gautschy, A., Ludwig, H. G., \& Freytag, B. 1996, A\&A, 311, 493

Goldreich, P., \& Keeley, D. A. 1977, ApJ, 211, 934

Pesnell, W. D. 1987, ApJ, 314, 598

Robinson, E. L., Kepler, S. O., \& Nather, R. E. 1982, ApJ, 259, 319

Unno, W., Osaki, Y., \& Ando, H. 1989, Nonradial Oscillations of Stars (Tokyo: Univ. Tokyo Press)

Winget, D. E., Van Horn, H. M., Tassoul, M., Hansen, C. J., Fontaine, G., \& Carroll, B. W. 1982, ApJ, 252, L65

Wu, Y. 1997, Ph.D. thesis, Caltech 